\title{
Spontaneous pneumoperitoneum associated with colonic pseudo-obstruction
}

\begin{abstract}
Pneumoperitoneum is almost always pathognomonic of a perforated abdominal viscus requiring urgent surgical intervention. Spontaneous or non-surgical pneumoperitoneum is a rare clinical condition arising secondary to abdominal, thoracic, gynaecologic or idiopathic causes. In addition to good clinical judgement, an important component in the management process is to rule out other causes of pneumoperitoneum by performing appropriate investigations. We describe a 60-year-old man who presented with clinical features of pseudo-obstruction, following an injury to his back which was compounded by hypokalaemia. Roentgenography revealed massive pneumoperitoneum and colonic distension. As there were no overt clinical features of peritonitis, the patient was managed conservatively with parenteral nutrition and close observation. A water-soluble contrast enema and computed tomography of the abdomen were of no help in identifying the cause of his pneumoperitoneum but were helpful in eliminating the presence of hollow viscus perforation or an obvious inflammatory focus. The aetiology of pneumoperitoneum in our patient was most likely due to dissection of air through the distended colonic wall, secondary to large bowel pseudo-obstruction. The diagnosis of spontaneous or non-surgical pneumoperitoneum is one of exclusion and we stress the importance of relying on clinical parameters when managing such patients conservatively.
\end{abstract}

Keyword: Pneumoperitoneum; Pseudo-obstruction 